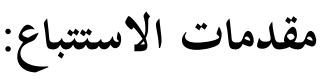

\section{الشرق موجود بغيره لا بذاته}

\author{
المؤلف: غريغوار منصور مرشو \\ الناشر : المعهد العالمي للفكر الإسلامي، (هيرندن، فرجينيا) \\ الطبعة: الأولى 1416هـ/1996م (160صفحة).
}

\section{بدران بن الحسن}

يعد هذا الكتاب الذي بين أيدينا حلقة مهمة في بناء منهجية التعامل مع الآخر الغربي، ذلك أنه يبحث في جذور الغرب الثقافية والمعرفية التي شكلت -ولا تزال- تعامُلَه مع غيره، كما يبرز الكتاب الاستعدادات الثقافية التي مكنت الغرب من أن يبلور، بوساطتها صورة عن ذاته، ويشكل صورة مشوهة للآخر لتأكيد ذاته، والتمركز المستعلي على غيره. ويتناول مصادر خطاب السيطرة على البشر بعد السيطرة على الطبيعة، ثم الاستشراق، وأيديولوجية الهيمنة.

يقع الكتاب في 160 صفحة من القطع المتوسط، متضمناً تصديراً للدكتور طه جابر العلواني، ومقدمة وأربعة فصول وخاتمة.

في التصدير أشار الدكتور العلواني إلى أن عنوان الكتاب "الاستباع" يستدعي إلى الذاكرة مجموعة من المفاهيم والمصطلحات سادت في أوساط مثقفي الأمة منذ منتصف القرن الماضي، مثل: الاستعمار، والاستشراق، والاستغراب، والاستكبار، ... النظام العالمي الجديد والإرهاب، والأصولية، والظلامية ونخوها. 
والكتاب يستدعي هذه المفاهيم لأنه يعالج "حقلاً معرفياً" واسعاً يتصل بكل هذه المفاهيم. وهذا الكتاب يتناول الغرب بتحليل بنائه وأطره المنهجية، ومسلماته المعرفية (الأبستمولوجية) وفلسفته ونظرياته وقواعده المعرفية، وينقب عن كيفية تحول الغرب إلى المركزية في رؤيته لذاته وتميشه للآخرين، بل واستتباعهم. ويشير إلى الطريقة التي تناول بها العقل المسلم ظاهرة الاستشراق، وأها كانت دون مستوى الإحاطة بالظاهرة، وانعدام الاهتمام بتحليل البنى المعرفية والأطر المنهجية للاستشراق بوصفه حقلاً معرفياً نشأ في إطار العلم الغربي وفلسفته ونظرياته وأسسه المعرفية، وكان موضوعه الآخر غير الغربي، مستمراً في الشرق المسلم، وأن هذا الحقل المعرفي قد أسس لنفسه علوماً لبناء شبكات في الشرق المسلم، وأن هذا الحقل المعريف قد أسس لنفسه علوماً لبناء شبكات المفاهيم الجلديدة والمنظومات المعرفية المؤطرة في فلسفته (التصدير، 10). في المقدمة يشير المؤلف عدة أفكار مهمة في سياق معالجته للموضوع، أهمها: - التناول الاجتزائي للاستشراق: فيرى الباحث أن الكتابات حول الاستشراق ونتائجه منذ عقدين من الزمن ونيف، وقع معظمها ضحية منظورات اجتزائية لم تضع الاستشراق ضمن النسق المعرفي المؤسسي العام للحضارة الغربية، فهو ليس ظاهرة موازية للنسق المعريف المؤسسي الغربي المهيمن ولا منقطعة عنه أو كأ عرضية فيه، إنما على العكس استمدت جذورها من هذا النسق بكل مكوناته المعرفية والمذهبية، نسجت خيوطها في كنفه، وتشكّل جزءاً أساسياً في إنشاء نموذج (الدولة-الأمة) في الغرب.

- طرق الاستعمار في بسط الهيمنة: إذ لم يلجأ الاستعمار - في فرض هيمنته ونمط إنتاجه وإملاء شروطه، بوصفها حقيقة وحيدة لازدهار الحضارة- دائماً إلى سياسة النهب الخالص وسياسة المدفع السافرة، إنما اعتمد على أساليب أخرى أيضاً في مرحلة ما قبل الاستعمار المباشر، وذلك عن طريق الاتفاقيات التجارية والعسكرية والعلمية مع حكام دول الأطراف وتكوين أنصار وزبائن مفتونين بمبادئه وقيمه ومؤسساته في المجتمعات المحلية، والتسلل إلى الضمائر وتطويعها وتسخيرها لصالحه.

- عقدة التفوق الغربي التي تحكم الغرب، إذ لكي يستأثر بزمام المبادر في معالجة التاريخ العالمي يرفض الاعتراف بالقيم والرموز الخاصة بالثقافات المغايرة، أو بفكرة تاريخ متعدد، ويلجأ إلى تقطيع مجتمعات 
الأطراف إلى شرائح وكيانات قبلية وطائفية، أو عرقية وإقليمية، ويحول تواريخ الشعوب إلى أصفار على هامش الحضارة، لا قيمة لها إلا بقدر اندماجها في دائرة السوق المتمم لحاجات إنتاجية المركز الأوربي.

- الجذور الأيديولوجية والمعرفية للغرب: فالجذور الأيديولوجية للغرب ونظريته المعرفية تأسست ابتداءً من عصر النهضة، ومشروع الغرب يتجه نحو تطوير قيم أخلاقية جديدة متحررة شيئا فشيئاً من الدين لصالح نظرية جديدة عن المعرفة، مثثلة في أولوية المعرفة الموضوعية المستمدة من مجالي التجريب والرياضيات.

- ذهنية الصراع والسيطرة على الطبيعة والإنسان التي تحكم الغرب في تعامله مع الشرق، ضمن تراتبية تنطوي على إقامة فوارق جوهرية ثابتة بينه وبين سكان الشرق، وجعل نفسه وصياً وحيداً في تقرير مصائرهم تحت شعار تحرير الوثنيين والكفار "الوحوش" أو "الهمج" من "الظلامية" و"العبودية."

بمقتضى هذه الاستراتيجية راح الغرب يُساوي نفسه مع التاريخ، وله وحده الاستحواذ على مواقع النجاح، ولو على حساب ثقافات وتواريخ الشعوب الأخرى ومحوها.

- نشأة (الأنثروبولوجيا) الغربية: فالغرب أنشأ هذا العلم لدراسة إنسان ما وراء البحار بمناهج جديدة تمدف إلى عزله عن كل الظروف الاجتماعية التاريخية ونكران ما يمثله من قيم ثقافية مغايرة. وحسب منظور (الأنثروبولوجيا) الغربية لم يعد ثمة شيء عقلاني، بالمعنى الحرفي سوى النظرية (الأنثروبولوجيا) "الإنسانية" عن ثقافة الشعوب التي اصطلح على تسميتها "بالبدائية" أو "الوحشية"، ولم يعد هناك من ثقافة سوى ثقافتهم

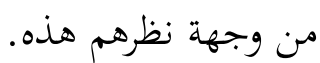

وإذا تم الاعتراف بعظمة حضارات الغير فبمقدار استجابتها لمصالح وترميزات المركزية الغربية. (ص 19

- (أيديولوجيا) الفتح، والتسويغ لخطاب السيطرة: إن الخطاب المسيطر والمؤسس في القرن التاسع عشر، تحالف تحت قيادته الدولة المركزية الدستورية والعلم، والجيش والصناعة والكنيسة حاملة رسالة التبشير، لتوزيع المهام وتقاسم النفوذ ضمن استراتيجية منظمة روج لها رواد (أيديولوجيا) الفتح أمثال مونتسكيو هيجل

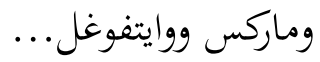


في الفصل الأول من الكتاب تناول الكاتب مظاهر نشوء النظام الغربي مركزاً على كيفية اشتغال القوانين الجديدة التي تحكمت في الحملات التبشيرية والمهام التي كلفت بها لغزو ما سُميّيَ بالشرق وتشويه صورته، ثم أبرز كيف انخرطت الدول الغربية، لحل مسائلها السياسية الداخلية، في الحملات التوسعية ما قبل الاستعمارية فيما وراء البحار، ثم كيف أسهمت في إضعاف الإمبراطورية العثمانية -التي كانت تشكل تحدّياً سافراً للغرب آنذاك- وترسيخ الانشقاقات الطائفية والعرقية والقبلية والإقليمية بين الجماعات المتعايشة في ظلها.

وفي خمسة نقاط يتناول النشوء والنماء الذي سار عليه النظام الغربي؛ ففي النقطة الأولى يتناول عملية التداخل بين السلطة الدينية للكنيسة مع الغزوات التجارية (المركنتيلية)، إذ ستتبلور (أيديولوجيا) تجعل من الربح قاعدة للالتقدم" ومن المبادلة الحرة أداة أكثر فعالية وضمانة لـ"الحضارة" وذلك حين تحولت التجارة عند التجار وأصحاب المصارف إلى "فضيلة" والمال إلى "صنم" بوصفهما شرطين أساسيين لضمان "النجاح" في الحياة العملية. (ص 28) (28). (2)

وفي مسار تشريع اللاهوت للناسوت "المركنتيلي" اضطرت كل الكنائس أن تتكيف مع التنمية الخارجية للروح التجارية أو التشجيع لها ثم الأخذ بالعقلية الصناعية... لقد أوجدت الرأسمالية روحها الخاصة، ووضعت الكنائس أمام ضرورة الاندماج بالقانون الطبيعي (للدولة-الأمة) (ص35-36). وفي النقطتين الثانية والثالثة: تناول الصراع الذي حدث بين الكنائس على الشرق ودخول البروتستانت حلبة المنافسة لماكان للمراسلين الكاثوليك من نشاط مكثف، وفي خِضَمّم هذا الصراع كان (protestant) نشر "الإميان المسيحي" يجري من خلال ثلاث قنوات: الصحافة والإرساليات ثم الطباعة لكوها وسيلة فعالة

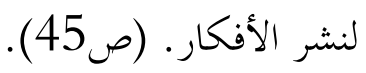
أما النقطة الرابعة فيتناول فيها اشتداد الهيمنة الأوروبية على الشرق الأوسط في مختلف المجالات في الثلاثينيات من القرن التاسع عشر، بخاصة في عهد محمد علي باشا إثر الشروع في "التنظيمات" الإصلاحية عام 1839م. 
وقد قدم المؤلف في هذا السياق حشداً من المعلومات عن الإرساليات الغربية وعن أنشطتها المتشبعة والفعالة. بين أهل الشرق، مبيناً ارتباطاتا المباشرة باستراتيجيات الدول الغربية.

وأما في النقطة الخامسة فقد تطرق الباحث إلى النتائج الضارة التي سبتها الإرساليات الأجنبية بمختلف أنشطتها سواء للمسلمين أو للمسيحيين الشرقيين أو غيرهم، إذ في إطار التوسع الاستعماري وترسيخ دعائم السيطرة الأوروبية في وعي الأهالي، خاصة باحتواء الأقليات الدينية والمذهبية والإثنية، تم استقطاها، ثم عزلها وجعلها معادية لقيمها ومحيطها الديني الثقافي والحضاري. وكانت أهم هذه النتائج الضارة تتمثل في: تأصيل الطائفية، وتفاقم الصراع بين الجماعات، وغرس المشروعين الانفصاليين الماروي،، واليهودي. فمن أجل تأصيل الطائفية، أنشأ النظام الغربي قاعدة للطائفية بزرعه لفكرة "الدولة - الأمة المجسد للطائفية نظاماً اجتماعياً، وقد ربطت هذه الدولة عمودياً بدول المركز وبعجلتها (nation-state) الاقتصادية، وقام بتلغيم القواسم المشتركة ووسع هوة الفوارق الاجتماعية وأثار النزعات الطائفية بين الجماعات الدينية والمذهبية والعرقية.

أما فيما يخص تفاقم الصراع بين الجماعات، فهو على ضربين: صراع بين الجماعات الدينية والمذهبية والعرقية، وصراع من مستوى آخر، وهو صراع النخبة المتغربة مع الأغلبية الشعبية.

لقد لاحظنا سابقاً ضمن استراتيجية التغلغل التبشيري، أن كل القوى الاستعمارية حاولت أن تدعم بطريقة منفردة أو مشتركة، جماعة على حساب جماعة أو جماعات أخرى، متعايشة سابقاً في ظل الخلافة العثمانية، من أجل مد هيمنتها وتكريسها على الخلافة العثمانية وتفكيكها، ثم الوصول إلى اصطناع دويلات مبنية على قواعد عرقية وطائفية وقبلية وإقليمية وجهورية. لكن المشروعين الذين استأثرا أكثر من غيرهما باهتمام القنصليات الأجنبية، في القرن التاسع عشر، هما إنشاء كيانين لليهود الأوروبيين والآخر للمسيحيين المحلين، وبالتحديد الموارنة، ليكونا منزلة حصنين متقدمين للمركزية الغربية في الشرق الأوسط ومنطلقين للمرافعة عن "العالم الحر.." 
وفي الفصل الثاني المعنون "من السيطرة على الطبيعة إلى السيطرة عل البشر" قام الكاتب بتحليل الكيفية التي تمت بها بلورة مفاهيم المركزية العرقية الغربية، وكيف تضافرت هذه المفاهيم مع حملات التبشير

$$
\text { باسم "العقل" و "العقلانية" أو "الموضوعية العلمية". }
$$

ولإبراز المعايير والقيم والعادات الذهنية التي تسكن الخطاب التاريخي المتعالي للنظام الغربي الصاعد، قام الباحث بمقاربة تلمس المقدمات والعناصر الإجرائية المنظمة و "المعقلنة" التي مكنت هذا النظام من تدعيم أسس الاستشراق، كعنصر مكتمل لهيمنته، وتبيان كيفية المحاججات "العقلانية" التي سمحت له بفرض ثقافته على الشعوب المهمشة وخاصة بعد "عصر الأنوار".

هذه المقاربة تناولها في عناصر أربعة: مصادر الخطاب الكوني في منعطف القرنين السادس عشر والسابع عشر، والعرقية المركزية الكامنة في الخطاب الكوني، والحروب بوصفها صمام أمان "للدولة-الأمة"، ثم استغلال أسطورة "الإنسان المتوحش الطيب".

فالمثقفون في أوروبا في بداية عصر النهضة انكبوا على استناق أنفسهم حول الأسباب التي تم إنشاؤها عن طريق الملاحظة الحسية من أجل توسع الحد الأقصى من فضاء السيطرة على الطبيعة. ومع توسع التجارة، وظهور علم (الأنثروبولوجيا)، وُلدت شبكة مؤسسية من المعارف السياسية و (الأيديولوجية)، فمكيافيلي عندما اكتشف التاريخ الروماني بحث فيه عن القوانين الأبدية لسيطرة البشر بعضهم على بعض، في كتابه "الأمير"، وظهرت نظرته في العصر التالي مع مذهب "المصلحة العليا للدولة" التي ما كان لها أن تقوم لولا تبرير وسائل السيطرة الوحشية...، فميكيافيلي بمبدأ "الغاية تبرر الوسيلة" الذي كان خلاصة كتابه أسس للغزو الاستعماري وأخلاقه النفعية.

أما هوبس فقد دفع هذا المفهوم إلى الحد الأقصى، واعتبر الحرية ليس شيئاً غير غياب كل ما يهول دون الحركة، كما أعلن أن على الدولة أن تضع الكنيسة والدين في خدمتها، ثم كوندورسيه، وديكارت الذي قدم حججاً (للإيديولوجية) الكاثوليكية وشجعها على تكييف لاهوقا مع التقدم والارتقاء الثقافي العقلاين بوجه كوني. 
ومع هيجل كشف الخطاب الكوني للغرب عن عرقيته المركزية الكامنة، ذلك أن هيجل لكي يدعم خطابه ويتفادى تحديد نفسه فقط بخطاب كوني متعارف عليه في عصره لجأ إلى التاريخ اليوناني الذي يعتبره المنطلق العام للتاريخ الإنساني برمته، وهو في أطروحته ينطلق من شقين: في الشق الأول يشيد بالتاريخ الغربي مرجعاً واحداً جديراً بالاعتبار. أما الشق الثاني فيحط من شأن تاريخ الحضارات المسماة بالشرقية ويعتبرها مرحلة زائلة.

فالجدلية الفكرية عند هيجل لا تتجاوز حدود الغرب، وكل فكر خارج هذه الحدود موسوم بالظلامية والاستبداد وانعدام الحرية والتاريخ.

هذه التعميمات تحولت إلى بداهات تعزز خطاب المركزية الغربية عن طريق "العلوم الإنسانية"، في إطار التطورية الداروينية- السبنسرية، والوضعية الأوغست-كونتية، ولا مادية التاريخية (ص 77-80).

ضمن هذا المنظور العام ستغدو كل المسوغات العقلانية ناجزة لشن الحملات والحروب على هذه الأمم الموسومة بـ "غير المتحضرة" وقد أصبحت، لاحقاً، حقاً قانونياً ومطلباً حيوياً "للدولة-الأمة "الغربية.

ولإنقاذ المجتمع المدني الغربي من الركود والموت يقترح هيجل سلسلة من العلاجات تنطوي على القيام بالحرب والاستعمار. فالحرب لها الفضل في إعادة بناء التجانس الوطني بتحاه التهديد مقبل من الخارج، والاستعمار سيسمح للدور الغنية بالتخلص من غير المرغوب فيهم.

أما أسطورة "الإنسان المتوحش الطيب" الموروثة عن العصور الوسطى وعصر النهضة فقد رسخت في الأذهان على شكل استيهامات غرائبية حتى بات الاستعمار يعد عملاً إنسانياً يسوغ غزو الشعوب "الوحشية" و"الكسولة"، أي: العاجزة عن الإنتاج واستغلال الثروات الطبيعية. الأمر الذي يشير شهوة التوسع والهيمنة عند الغزاة.

أما الفصل الثالث فقد تناول فيها الاستشراق و(أيديولوجية) الهيمنة: فلكي نُضفي الدوائر الاستعمارية على (أيديولوجيتها) التوسعية صبغة قانونية وعقلانية كان عليها أن تقدم علومها في بجال الإنسان بصفة علوم حيادية عالمية شبيهة بالعلوم الطبيعية... ولم تتردد "العلوم الإنسانية" في توظيف أسطورة الإنسان 
المتوحش" بما يخدم مصالح دولها. والقصد ليس بتريده من مزاياه الفكرية فحسب، إنما هو تأسيس خطاب علمي خخص للآخرين يبرز للمركزية العرقية الغريبة زعزعة ثقة الشعوب الشرقية بذاتا وبمعاييرها وتدمير بجتمعاها وعوامل الاستمرارية عندها، ولعل هذا ما جعل العلماء على الرغم من اختلاف مشاربمم (الأيديولوجية) )يجمعون على إسقاط أحكام معيارية مسبقة الصنع على الشرق وإلصاق كل النعوت السلبية به: من قبيل الاستبداد، والتأخر، والكسل، واللاعقلانية، واللاتاريخية.

من أجل "البحث عن آدم جديد" للأوروبي الأبيض والمتفوق والعنصري، عمل الاستشراق على خطين: الوعي واللغوي بمنظار أوروبا ومقاييسها، والميل إلى التجزئة والتفريع وإعادة التجزئة لمباحثه دون أن يغير من رأيه حول الشرق.

لقد بدا العرق واللغة مرتبطين بطريقة مبهمة عند المستشرقين، وصار الشرق "الخير" حقبة كلاسيكية مضت، موضوعة في مكان ما من الهند منذ زمن سحيق، بينما الشرق "السيء" ما يزال بجوهره في آسيا اليوم وفي أقسام من إفريقيا الشمالية، وفي كل مكان حيث يوجد الإسلام.

أما الحتمية العرقية التي غجها علماء اللسانيات والسلالات فقد أدت إلى جعل العرق صنماً محركاً للتاريخ على يد شارك كونت، وفيكتور دوليل، وغوبينو الذي يرى أن العرق الأبيض يظهر فيه بوضوح الجمال والذكاء والقوة، ويمتلك عنصرين أساسيين لكل حضارة هما: دين وتاريخ، فضلاً عن اجتماعيته المضرة وتفاوته وتوسعه عن طريق الغزو (ص 95).

أما أرنست رينان فيرى أن العرق السامي يعتبر شكلاً منحطاً ذا تركيب أدنى من الطبيعة الإنسانية بالمعنى الأخلاقي والبيولوجي (ص 96)، يستثنى اليهود بإدراجهم في دائرة المركزية العرقية الغربية بدعوى أن

$$
\text { العرق "الإسرائيلي" قدم للعالم أكبر الخدمات العظيمة (ص 97) }
$$

والحنط نفسه سار عليه كوفييه، وسانت فانسان، وكاتروفاج، ودوبلاج في فرنسا وتوما أرنولد، وكنوكس وداروين وسبنسر في بريطانيا ثم في الولايات المتحدة على يد ميرتون، وجيرون، ونوت من دعاة الأشكال 
المتطرفة للنظرية العنصرية المعادية للسود، وبلغ الخطاب العرقي العنصري على يد علماء الأناسة الألمان ابتداء من القرن التاسع عشر حداً متطرفاً مع مبدأ تفوق العرق الجرماني.

وتحت قيادة هذا الخطاب "العلمي" المسيطر والمؤسس في القرن التاسع عشر تحالفت الدولة الممركزة الدستورية، والعلم، والجيش، والصناعة والكنيسة لتوزيع الأدوار وتقاسم المهمات ضمن استراتيجية منظمة، تراوحت مهماتما حسب الظروف، بين مطاردة السكان المحليين وتميشهم وبين تسخيرهم وتصفيتهم. كل ذلك باسم الحضارة والحرية وحقوق الإنسان، وتقدم الإنسانية. هذا التقدم: (الاستعمار والتحضير) نظر له كل فلاسفة أوروبا من (أوغست كونت)، وهيجل، إلى المدرسة الماركسية التي لم تفلت من الأسطورة الخاصة بالتقدم الأحادي والحتمي للإنسانية، ولم ينقطعا معرفياً ولا إيديولوجياً مع ثوابتهما الداعية إلى رسالة حضارية في الشرق، وفي الحقيقة ما قامت به الماركسية في أدبياتا حول الشرق كان امتداداً للمدرسة الاستشراقية (الليبرالية).

وخلاصة الكلام حول الاستشراق و(أيديولوجية) الهيمنة التي أنتجها أن ما يريده المنظرون والمستشرقون هو وضع شعوب الشرق أمام خيارين لا ثالث لهما، إما الرضوخ النهائي لمنطق الغرب، وإما أغا ستظل تعتبر، من قبل الخارج، محكومة بعدم قدرتا على مواجهة الحياة.

وأما في الفصل الأخير فقد تناول المؤلف "حملة بونابرت على مصر" التي هي نموذج تطبيقي للأدوات والترميزات والتصورات الغرائبية التي يحملها الغرب نحو الشرق.

إذ الحملة على مصر -حسب رأي الكاتب- لم تكن ثمرة مشروع عرضي من بنات فكر نابليون أو عبقريته الأسطورية، إنما جاءت نتيجة تراكم لكل المشاريع السابقة التي كانت متداولة فيما وراء الكواليس، وما كان نابليون فيها إلا الأداة الجامعة المنظمة والمنفذة، خدمة للمصالح العليا للدولة الفرنسية. كان بونابرت ينوي إلحاق الإسلام بالمركزية الأوروبية ومن أجل تحقيق هدفه حاول بث التفرقة العرقية بين المماليك والمصريين من جهة، وبين العرب الاجتماعي والعثمانيين من جهة ثانية باسم الإسلام، ثم تعميق 
الشقاق والصراع الاجتماعي ما بين الأديان والمذاهب باسم تقديم حلول علمانية زمنية مستمدة من مبادئ عصر الأنوار، للأقليات الدينية والمذهبية.

استخدم نابليون مجموعات من النخب المحلية لضمان سريان أوامره، وعمل على تأييد المارونيين، واستعطف اليهود ودعاهم إلى النهوض لتحقيق مطالبهم والاستغلال الفرصة للعودة إلى أرض الميعاد.

ورغم أن احتلال بونابرت لمصر باء بالفشل على الصعيد العسكري، إلا أن آثاره لا تزال حتى اليوم تسيطر على منظوراتنا الثقافية والسياسية، ولقد انقسمت غالبية النخب المحلية المحدثة إزاء هذه الحملة إلى ابحاهين: الأول رأى فيها منعطفاً حاسماً في تشكل تاريخهم الحديث، لكوها كانت مبعثاً على "النهضة العربية" بفعل آليات المثاقفة مع الآخر الغربي. أما الثاني: فيتمثل في التوفيق ما بين العلوم الحديثة المستجلبة والتراث العربي الإسلامي، وذلك بتطويعه للنموذج الغربي.

ولا يزال الصراع يدور بشكل سحالي دون نقاط مضيئة حول الأسس المشتركة لتحقيق النهضة.

في الخاتمة يناقش الباحث فكرة بحاوز الغرب لمقولاته المعرفية، غير أن الباحث -وأظنه محقاً في ذلكيرى أن لا نستعجل ونعمم الحكم على غالبية (الأنثروبولوجيين) والمؤرخين والمستشرقين بأفم قد قطعوا، من حيث المضمون، مع مركزية النظام الغربي لكوفم انتقدوه أو انتقدوا مظاهره.

ذلك لأن عملية القطيعة تستدعي إعادة نظر جذرية في العديد من المسلمات الغرائبية والاستيهامية الثاوية في النظام المعريف الغربي المؤسسي، ولا يكفي أن تطلق صفة القطيعة حتى يسلم آلياً بكل أشكال التطبيقات المعرفية وما تتضمنه من إسقاطات ماسخة على شعوب الشرق وثقافاته.

إن أي ترويج اعتباطي لمفهوم القطيعة -دون قيام نوع من علاقة المحاورة المتفاعلة بين الغربي والطرف الآخر - يعتبر إضافة أيديولوجية إلى جملة ما هو سائد.

هذا وإن الكتاب مليء بالأفكار يمتاج إليه كل مثقف يريد لنفسه أن يتفاعل إيجابياً مع الغرب المهيمن بخيله ورجله وأفكاره وإنتاجه، ذلك أنه يبحث في الجذور المعرفية التي أسست للخطاب الغربي المستعلي، ويقوم بالحفر في عمق مدلولات الخطاب ليبزها، ويناقش "حقلاً معرفيا" يجتاج إلى ارتياد الكثير 


$$
\text { وتن ذوي البصائر وأولي الألباب، حتى يؤسس التعامل مع الغرب على علم، وتختصر الكثير من المعارك الوهمية }
$$

